\title{
STOCHASTIC PERTURBATIONS TO CONSERVATIVE DYNAMICAL SYSTEMS ON THE PLANE. I: CONVERGENCE OF INVARIANT DISTRIBUTIONS
}

\author{
G. WOLANSKY
}

\begin{abstract}
We consider a nonlinear system on the plane, given by an oscillator with homoclinic orbits. The above system is subjected to a perturbation, composed of a deterministic part and a random (white noise) part. Assuming the existence of a finite, invariant measure to the perturbed system, we deal with the convergence of the measures to a limit measure, as the perturbation parameter tends to zero. The limit measure is constructed in terms of the action function of the unperturbed oscillator, and the strong local $L_{2}$ convergence of the associated densities is proved.
\end{abstract}

\section{Introduction. Let}

$$
\dot{z}=\mathbf{b}(z)
$$

be a dynamical system on $\mathbf{R}^{2}$, where $\mathbf{b}$ is a divergence-free vector field. Assume, moreover, that $\mathbf{b}$ is representable by a single-valued Hamiltonian $H$ :

$$
\mathbf{b}=\bar{\nabla} H \equiv\left(-\frac{\partial}{\partial x_{2}} H, \frac{\pi}{\partial x_{1}} H\right), \quad\left(x_{1}, x_{2}\right) \equiv z \in \mathbf{R}^{2} .
$$

Consider now (1.1) with an initial probability distribution given by $d \mu^{0}=\rho^{0} d \mu^{L}$, where $\rho^{0}$ is a differentiable density function and $\mu^{L}$ is the Lebesgue measure on $\mathbf{R}^{2}$. The time evolution of $\mu$ is given by the Liouville equation

$$
\partial \rho / \partial t+\partial_{H} \rho=0, \quad \rho(0)=\rho^{0}
$$

with

$$
\partial_{H} \equiv \bar{\nabla} H \cdot \nabla .
$$

A stationary density distribution of $(1.1)$ is given by a stationary solution to $\left(1.1^{\prime}\right)$, i.e. $\partial_{H} \rho=0$. It is evident that there is an infinite number of stationary densities.

Let us consider a perturbation to (1.1)

$$
\dot{z}=\mathbf{b}(z)+\varepsilon Q(z)+\sqrt{\varepsilon} \dot{W},
$$

where $W$ is a two-dimensional Brownian motion, $Q$ is a deterministic vector field on $\mathbf{R}^{2}$ and $\varepsilon>0$. Scaling $t$ by $\tau=\varepsilon t,(1.3)$ presents a diffusion process on $\mathbf{R}^{2}$, whose generator is given by

$$
L^{\varepsilon}=\frac{1}{\varepsilon} \partial_{H}+\Delta+Q \cdot \nabla .
$$

Received by the editors July $15,1987$.

1980 Mathematics Subject Classification (1985 Revision). Primary 35B20, 35R60, 60J60; Secondary 35B45.

Key words and phrases. Diffusion process, perturbation, invariant distribution. 
If (1.3) is positively recurrent for some $\varepsilon>0$, then the process is ergodic (cf. [6]). In that case, there exists a finite invariant distribution $\mu^{\varepsilon}=\rho^{\varepsilon} d \mu^{L}, \mu^{L}$ the Lebesgue measure on $\mathbf{R}^{2}$ (cf. [4]), where

$$
\left(L^{\varepsilon}\right)^{*} \rho^{\varepsilon}=0 .
$$

Here $L^{*}$ is the formal adjoint of $L$.

Our interests are the following:

(a) Under which conditions on $H$ and $Q$ is the process (1.3) positively recurrent for $\varepsilon>0$ small enough?

(b) Assume (1.3) is positively recurrent for $\varepsilon>0$ small enough. Under which conditions does

$$
\lim _{\varepsilon \rightarrow 0} \mu^{\varepsilon}=\mu^{0}
$$

exist, where $\mu^{0}$ is a finite probability measure on $\mathbf{R}^{2}$ ? What is the nature of the above convergence, and how does $\mu^{0}$ depends on both $H$ and $Q$ ?

On an intuitive ground, we expect that, if (1.6) holds, then $\mu^{0}$ is an invariant distribution of the unperturbed process (1.1). If, in particular, $\mu^{0}$ admits a differentiable density $\rho^{0}$, then

$$
\partial_{H} \rho^{0}=0 .
$$

Thus, if (1.6) holds, we may single out a stationary distribution of (1.1) which is consistent with the given forcing $Q$. The convergence of (1.6) may be obtained from the convergence of the semigroups $T^{\varepsilon}$ associated with the diffusion process, together with some assumptions (see below).

PROPOSITION 1.1. Assume

$$
\lim _{\varepsilon \rightarrow 0} T_{t}^{\varepsilon}(\varphi)=T_{t}^{0}(\varphi) \quad \forall \varphi \in C^{0}\left(\mathbf{R}^{2}\right), t \in \mathbf{R}^{+},
$$

where the convergence above is uniform on each compact. Assume $\mu^{\varepsilon}$ is an invariant measure w.r. to the process $T^{\varepsilon}$ and let $M$ be the set of weak limits of $\mu^{\varepsilon}, \varepsilon \rightarrow 0$. Let $K$ be the set of invariant measures w.r. to $T^{0}$. Then $M \subset K$.

Proposition 1.1 is a special case of Lemma 4 in [7]. Assume the conditions of this proposition. If, in addition (1.3) is positively recurrent for $\varepsilon>0$ small enough, $\left\{\mu^{\varepsilon}\right\}_{\varepsilon>0}$ is relatively compact in the weak topology and $T^{0}$ admits a unique invariant measure, then we obtain the weak convergence in (1.6). Considering (1.3) with (1.2) it seems natural to apply formal averaging to deduce the convergence (1.8). In fact, we may single out any function of $H$ as a slow variable and $x_{2}$, say, as a fast variable, and obtain the limiting diffusion as a function of $H$ by averaging over the fast variable. Using the averaging method, Proposition 1.1 and the assumption thereafter, Khas'minskii [8] obtained the weak convergence of (1.6) for $H$ given by an harmonic oscillator. In a subsequent paper [9], he extended the proof to a general Hamiltonian, provided the frequency of oscillations of the unperturbed system (1.1) never vanishes. In that case, he got the limiting distribution $\mu^{0}$ in terms of a density $\rho^{0}=\rho^{0}(H)$.

If (1.1) admits orbits of zero frequency, the averaging method cannot be fully justified. In particular, assume that (1.1) contains a homoclinic orbit. In that case, 
$\iota$ pair of slow-fast variables does not globally exist and (1.3) cannot be transformed, even formally, to a system compatible with the usual averaging principle (see §2). This is not just a technical difficulty, but an essential one, as homoclinic orbits separate, in general, different modes of oscillations, so the future evolution of the diffusion orbit is very sensitive near those particular orbits.

One may attempt to overcome this difficulty by using abstract homogenization theorems which do not depend on a special coordinate representation. Kurtz [10] had presented a convergence theorem of semigroups generated by the operators

$$
L^{\varepsilon}=\varepsilon^{-1} B+A
$$

where $A, B$ generate strongly continuous, contraction semigroups, and both admit a common core. Let $P$ be the projection operator

$$
R(P)=N(B), \quad R(B) \text { is dense in } N(P) .
$$

Assume $L^{0}=P A P$ is a generator of a strongly continuous semigroup $T_{t}^{0}$ on $R(P)$. If $T^{\varepsilon}(t)$ is the semigroup generated by $L^{\varepsilon}$, then

$$
\lim _{\varepsilon \rightarrow 0} T^{\varepsilon}(t) \varphi=T^{0}(t) \varphi, \quad \varphi \in R(P),
$$

holds in the space norm uniformly w.r. to $t$ on bounded intervals [10, Theorem 2.1].

In order to apply Kurtz's theorem together with Proposition 1.1 to the present case, we have to use the norm of uniform convergence, or at least a norm equivalent to uniform convergence on compacts. In the present case, $B \equiv \partial_{H}$ and a candidate for the projection $P$ is given by the limit

$$
P \varphi=\lim _{\lambda \rightarrow 0} \lambda \int_{0}^{\infty} e^{\lambda t} S_{t} \varphi d s
$$

where $S_{t}$ is the semigroup generated by $B$, i.e., the time shift operator along the orbits of (1.1). If $H$ contains a homoclinic orbit and $\varphi$ is a bounded, continuous function, then the r.h.s. of (1.12) fails, in general, to converge in the uniform topology since $P \varphi$ may admit a discontinuity along a saddle connection of $H$. Thus (1.10) is not satisfied, in general, for $L^{\varepsilon}$ defined by (1.4) on $C^{0}\left(\mathbf{R}^{2}\right)$ with the associated sup norm. If we consider $L^{\varepsilon}$ on $L_{2}\left(\mathbf{R}^{2}\right)$, say, then (1.12) holds in the above norm and (1.10) is satisfied [5, p. 516]. In this case, however, the limit in (1.11) holds in $L_{2}$, rather than uniformly, and we cannot apply Proposition 1.1 unless some a priori estimate on $\mu^{\varepsilon}$ is known in advance. Even so, the convergence in (1.11) holds only on $R(P)$, so the best we can get out of Kurtz's theorem and a possible generalization of Proposition 1.1 is the weak convergence of the marginals of $\mu^{\varepsilon}$ with respect to the $\sigma$-algebra generated by all functions in $R(P)$ (in our case the minimal $\sigma$ algebra generated by $H$ ). In order to prove the weak convergence in (1.6) we have to remove this conditioning.

Another approach to the convergence of the diffusions is due to the martingale formulation of the process. Such an attempt was carried out by R. Pinsky [11] and $\mathrm{M}$. Berger [2]. The above approach leads to a weak convergence of the martingale solution of (1.3), and hence to the weak convergence of $\mu^{\varepsilon}$ on marginals. The weak convergence of $\mu^{\varepsilon}$ is evaluated by proving asymptotic independence, at $\varepsilon \rightarrow 0$, of the marginals of $\mu^{\varepsilon}$ with respect to the $\sigma$-algebras generated by $N(P)$ and $R(P)$, respectively. 
In the present paper we are dealing directly with the convergence of the invariant measures, while the convergence of the diffusion process is out of scope. We impose the following standing assumption:

ASSUMPTION A. 1. The process (1.3) is positively recurrent for each $\varepsilon>0$.

2. The set of invariant measures $\left\{\mu^{\varepsilon}\right\}, \varepsilon>0$, associated with (1.3) is relatively compact in the weak topology of measures.

In a companion paper [12] we introduce and prove sufficient conditions for Assumption A. Here we take Assumption A for granted and prove the convergence (1.6). The conclusions are stronger than in the previous approach (strong $L^{2}$ convergence vs. weak convergence of (1.6)) and rather general (existence of saddle connections is permitted). The method is also applicable to nonlinear systems with multiple frequencies, although the extension is nontrivial. We will deal with multiple-frequency systems in a separate paper [13].

Our approach is based on a representation of an invariant measure to a diffusion process due to Khas'minskii [6]. Let $\gamma_{1}$ be a closed counter in the phase space, and let $\gamma_{2}$ be a second counter, contained in the interior of $\gamma_{1}$. Define the Markov time due to a diffusion process parametrized by $\varepsilon$ :

$$
\tau^{\varepsilon}=\left\{\inf t ; z^{\varepsilon}(0) \in \gamma_{1}, z^{\varepsilon}(t) \in \gamma_{1} \text { and } \exists 0<s<t, z^{\varepsilon}(s) \in \gamma_{2}\right\}
$$

where $z^{\varepsilon}$ is a realization of the process.

Then the diffusion is positively recurrent if

$$
\sup _{y \in \gamma_{1}} E_{y} \tau_{1}^{\varepsilon}<\infty
$$

Since the diffusion is nondegenerate and all coefficients are smooth, (1.14) is equivalent to

$$
\sup _{y \in \gamma_{2}} E_{y} \tau_{1}^{\varepsilon}<\infty
$$

where $\tau_{1}^{\varepsilon}$ is the hitting time of $\gamma_{1}$ (cf. [3]).

Assume the process is recurrent. Then, one can define a stationary Markov chain on $\gamma_{1}$ by realizations:

$$
z^{\varepsilon}(0) \rightarrow z^{\varepsilon}(\tau) \quad \forall z(0) \in \gamma_{1} .
$$

Since the diffusion is nondegenerate and $\gamma_{1}$ is compact, the above chain admits a unique invariant distribution $\nu^{\varepsilon}$. Then, the invariant measure $\mu^{\varepsilon}$ of the diffusion process is representable by

$$
\int \varphi d \mu^{\varepsilon}=C^{\varepsilon} \int_{\gamma_{1}} E_{y}^{\varepsilon}\left(\int_{0}^{\tau^{\varepsilon}} \varphi\left(z^{\varepsilon}(s)\right) d s\right) \nu^{\varepsilon}(d y)
$$

where $C^{\varepsilon}$ is a normalization constant given by

$$
C^{\varepsilon}=\left[\int_{\gamma_{1}} E_{y}^{\varepsilon}\left(\tau^{\varepsilon}\right) \nu^{\varepsilon}(d y)\right]^{-1}
$$

and $\varphi$ is any continuous bounded function (cf. [6]).

From (1.15) it turns out that the positive recurrency and weak compactness of the invariant measures can be proved by estimates on $\tau_{1}^{\varepsilon}$. This is gained by 
constructing barrier functions on the external domain $\Omega_{1}$ with the boundary $\gamma_{1}$ (see $[3])$.

In order to show the weak convergence of $\mu^{\varepsilon}$ on some compact $K \subset \mathbf{R}^{2}$, we have to show the convergence of the r.h.s. of (1.17) given an arbitrary $\varphi \in C_{0}^{\infty}\left(\mathbf{R}^{2}\right)$, supported in $K$. In order to show strong $L^{2}$ convergence of $\mu^{2}$ on $K$, the convergence of (1.17) has to be uniform with respect to any $\varphi$ supported in $K$ in the unit ball of $L_{2}\left(\mathbf{R}^{2}\right)$. Now, if $K$ is included in the interior of $\gamma_{1}$, we may replace $\tau^{\varepsilon}$ by $\tau_{2}^{\varepsilon}$ in the integral of (1.17), where $\tau_{2}^{\varepsilon}$ is the hitting time of $\gamma_{2}$. Thus, the integral of (1.17) is given, in this case, by

$$
C^{\varepsilon} \int_{\gamma_{1}} E_{y}^{\varepsilon}\left(\int_{0}^{\tau_{2}^{\varepsilon}} \varphi\left(z^{\varepsilon}(s)\right) d s\right) \nu^{\varepsilon} d(y)=C^{\varepsilon} \int_{\gamma_{1}} U_{\varphi}^{\varepsilon}(y) \nu^{\varepsilon}(d y)
$$

where $U_{\varphi}^{\varepsilon}$ solves the Dirichlet problem in the bounded domain $\Omega_{2}$ with boundary $\gamma_{2}$ :

$$
L^{\varepsilon} U_{\varphi}^{\varepsilon}=-\varphi,\left.\quad U_{\varphi}^{\varepsilon}\right|_{\gamma_{2}}=0
$$

(see [4]). Consequently the convergence of $\mu^{\varepsilon}$ on compacts is related to the convergence of the solutions of a Dirichlet problem on bounded domains, while the question of positive recurrency is related to estimates of barrier functions on an external domain. The first question is treated in $\S 3$ (Theorem 2.1, §2), and the second in [12]. In $\S 2$ we compute explicitly the limit distribution $\mu^{0}$ and state our assumptions and main results.

2. Main results. Let $\mathbf{b}(1.1)$ be represented by a single-valued Hamiltonian $H$.

Assumption B. $H$ is $C^{\infty}$ and admits a finite number of critical points. All local maxima and minima of $H$ are nondegenerate.

Assumption B permits us to deal with rather general flows of (1.1) on a bounded domain. In fact, it can be generalized a great deal. On the other hand, no critical points of $H$ exist in a neighborhood of infinity.

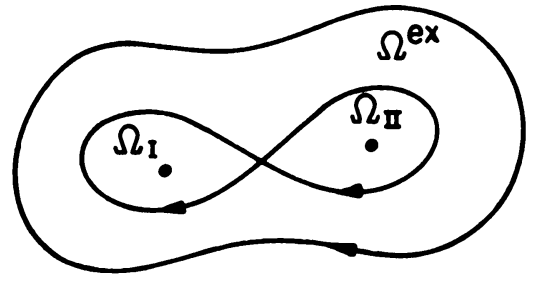

a

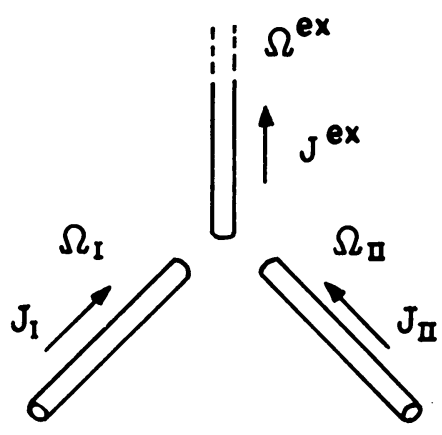

b 
From B we conclude that the phase space $\mathbf{R}^{2}$ is composed of a finite number of bounded domains of oscillations, $\Omega_{\alpha}$, and a single unbounded domain $\Omega^{e x}$. The boundary of each domain is composed of saddle connections (separatrix), i.e. level curves of $H$ which connect its saddle points. Each bounded component $\Omega_{\alpha}$ contains a unique center of oscillations $z_{\alpha}$, which is either a local minima or maxima of $H$, while $\Omega^{e x}$ contains no critical point of $H$. In Figure 1a we see a typical example, with $\Omega_{\alpha} ; \alpha=I, I I$ are the bounded domains associated with two different modes of oscillations. Here, $z_{0}$ stands for the saddle point.

Each domain of oscillation can be mapped into a cylinder by a pair of canonical variables $(J, \theta), J \in \mathbf{R}^{+}, \theta \in S^{1}$. The action $J_{\alpha}=J_{\alpha}(H)\left(J^{\epsilon x}=J^{e x}(H)\right)$ is defined by the area enclosed inside the contour $H=$ const.

$$
J(H)=\oint_{H(z)=H} x_{1} d x_{2} .
$$

We shall use $J(z) \equiv J(H(z))$ and $J=J(H)$ freely in the text. Since $H=H(J)$, the dynamical system (1.1) takes the simple form

$$
\frac{d J}{d t}=-\frac{\partial H}{\partial \theta}=0, \quad \frac{d \theta}{d t}=\frac{\partial H}{\partial J} \equiv \omega(J),
$$

where $\omega(J)$ is the frequency of oscillations (cf. [1]). Thus, the phase space $\mathbf{R}^{2}$ is decomposed into a finite set of finite cylinders, and a single, semi-infinite cylinder, associated with the unbounded domain of oscillation (cf. Figure 1b). By (1.2), $J_{\alpha}\left(z_{\alpha}\right)=0$. The upper boundary of a finite cylinder is mapped into a part of the separatrix. Let $\bar{J}_{\alpha}$ be the area of $\Omega_{\alpha}$. Then $J_{\alpha}=\bar{J}_{\alpha}$ on the upper boundary of each finite cylinder. The lower boundary of the semi-infinite cylinder is also mapped into the separatrix, and we define

$$
J^{e x}=\bar{J}^{e x}=\sum_{\alpha} \bar{J}_{\alpha}
$$

at the bottom of the semi-infinite cylinder, where $\alpha$ runs over all finite cylinders, in consistency with the area interpretation.

As a particular example, consider $H$ of the form

$$
H\left(x_{1}, x_{2}\right) \equiv \frac{1}{2} x_{1}^{2}+U\left(x_{2}\right) .
$$

If the potential $U$ is quadratic

$$
U=\frac{1}{2} \omega^{2} x_{2}^{2}
$$

where $\omega=$ const, then

$$
J=\frac{H}{\omega}=\frac{1}{2}\left(\frac{x_{1}^{2}}{\omega}+\omega x_{2}^{2}\right)
$$

is defined globally. There is no separatrix and the plane is mapped onto a single, semi-infinite cylinder. Notice that, in this case

$$
|\nabla J|(z)=O\left(J^{1 / 2}(z)\right)=O(|z|)
$$

and $\Delta J$ is a constant. In the general case we conclude from Assumption B that $J$ is approximated by a definite quadratic form near each center of oscillation. Hence (2.6) is satisfied in a neighborhood of $z_{\alpha}$ and

$$
\lim _{z \rightarrow z_{\alpha}} \Delta J=\text { const }>0
$$

for each bounded cylinder $\Omega_{\alpha}, J=J_{\alpha}$. 
Given a function $\sigma$ on $\mathbf{R}^{2}$, we define $\langle\langle\sigma\rangle\rangle$ as a function on $\mathbf{R}^{2}$ by

$$
\langle\langle\sigma\rangle\rangle(z)=\iint_{S(z)} \sigma
$$

where $S(z)$ is the bounded domain enclosed by the connected component of the level curve of $H=H(z)$. It is evident that $\langle\langle\sigma\rangle\rangle$ is not necessarily a continuous function, even if $\sigma$ is. For smooth $\sigma,\langle\langle\sigma\rangle\rangle$ admits, in general, a jump discontinuity across the separatrix, and is smooth on $\Omega^{e x} \cup_{\alpha} \Omega_{\alpha}$. Define

$$
\psi=\frac{\langle\langle\nabla \cdot Q\rangle\rangle}{\langle\langle\Delta J\rangle\rangle}
$$

The definition of $\langle\langle\Delta J\rangle\rangle$ is not completely clear since $\Delta J$ generally blows up near a separatrix. We understand $\langle\langle\Delta J\rangle\rangle$ by application of the divergence theorem, as if $J$ is smooth:

$$
\langle\langle\Delta J\rangle\rangle(z)=\oint_{\gamma(z)} \frac{\partial J}{\partial n} d \Gamma
$$

where $\gamma(z)$ is the counter of $J=J(z)$, and $J$ is given by $J_{\alpha}, J^{e x}$ on each $\Omega_{\alpha}$ and $\Omega^{e x}$, respectively. Notice that Assumption B guarantees the positiveness of $\Delta J$ at the centers of oscillations ${ }^{1}$ (cf. (2.7)). Thus

$$
\lim _{J \rightarrow 0} \psi=\left.\frac{\nabla \cdot Q}{\Delta J}\right|_{z=z_{\alpha}} \neq \pm \infty
$$

for any $J=J_{\alpha}$ in the bounded domain $\Omega_{\alpha}$. Define

$$
\begin{aligned}
& \chi(z)=\iint_{J(z) \leq J \leq \bar{J}} \psi ; \quad z \in \Omega_{\alpha}, \\
& \chi(z)=\iint_{\bar{J} \leq J \leq J(z)} \psi ; \quad z \in \Omega^{e x},
\end{aligned}
$$

where $\bar{J}$ stands for $\bar{J}_{\alpha}, \bar{J}^{e x}$ respectively, by the notation of (2.4). Notice that $\chi$ is a continuous function on $\mathbf{R}^{2}$ and $\chi=0$ on the whole separatrix. Define now a measure $\mu^{0}$ given by the density $\rho^{0}$ :

$$
\rho^{0}=C e^{\chi}, \quad \iint \rho^{0} d x_{1} d x_{2}=1 .
$$

with $C$ as a normalizing factor. The integrability of $\rho^{0}$ is assumed. (This is a direct consequence of the sufficient conditions to Assumption A introduced in [12].) Define

$$
\langle\sigma\rangle(J)=\frac{d}{d J}\langle\langle\sigma\rangle\rangle, \quad J \neq \bar{J}_{\alpha}, \bar{J}^{e x}, \quad .
$$

where $\langle\langle\sigma\rangle\rangle$ is taken as a function of $J$. We call $\langle\sigma\rangle$ the average of $\sigma$. Notice that $\langle\sigma\rangle$ is, in general, discontinuous across the separatrix. It is exactly the projection operator $P$ defined in (1.12). An equivalent definition is given by

$$
\langle\sigma\rangle(z)=\oint_{\gamma(z)} \frac{\sigma\left(x^{\prime}\right)}{\left|\nabla J\left(z^{\prime}\right)\right|} d \Gamma\left(z^{\prime}\right)
$$

\footnotetext{
${ }^{1}$ It was pointed to me by M. Berger that $\underline{\lim } \Delta J>0$ at any minima (maxima) of $H$, wherever degenerate or not. However, $\Delta J$ may be infinite in degenerate cases.
} 
where $\langle\sigma\rangle(z)=\langle\sigma\rangle(J(z))$. Using (2.14) and the divergence theorem, we obtain

$$
\langle\nabla J \cdot \eta\rangle=\langle\langle\nabla \cdot \eta\rangle\rangle
$$

for any vector field $\eta$ and $J \neq \bar{J}$. Letting $\eta=Q$ and $\eta=\nabla J$ in (2.15), we may write (2.9) equivalently as

$$
\psi=\langle\nabla J \cdot \nabla Q\rangle /\left\langle|\nabla J|^{2}\right\rangle .
$$

THEOREM 2.1. Under Assumptions $\mathrm{A}$ and $\mathrm{B}$, there exists a unique limit

$$
\lim _{\varepsilon \rightarrow 0} \mu^{\varepsilon}=\mu^{0}
$$

where $\mu^{0}$ is given in terms of the density $\rho^{0}(2.13)$, and the limit (2.15) is the strong limit in the $L_{2}^{\mathrm{Loc}}\left(\mathbf{R}^{2}\right)$ sense (i.e. the densities associated with $\mu^{\varepsilon}$ converge strongly in $L_{2}^{\mathrm{Loc}}$ ).

In particular, we conclude that $\left\{\mu^{\varepsilon}\right\}$ admits a density $\rho^{\varepsilon}$ in $L_{2}^{\text {Loc }}\left(\mathbf{R}^{2}\right)$, uniformly bounded in the norm w.r. to $\varepsilon>0$ on each compact.

In the next section we prove Theorem 2.1. By (1.20) and the remark thereafter, it is related to estimates of the solutions to the Dirichlet problem on bounded domains.

3. Convergence in the internal domain. Define a contour $\gamma_{J}^{\alpha}\left(\gamma_{J}^{e x}\right)$ in $\Omega_{\alpha}$ $\left(\Omega^{e x}\right)$ as

$$
\gamma_{J}=\{z ; J(z)=J\}
$$

where $\gamma_{J}=\gamma_{J}^{\alpha}\left(\gamma_{J}^{e x}\right)$ for $J(z) \equiv J_{\alpha}(z)\left(J^{e x}(z)\right)$, respectively, and $J>0$ is a fixed constant. Given $J_{i}>\bar{J}^{e x}, i=1,2$ (see 2.3), define $\gamma_{i}=\gamma_{J_{i}}^{e x} i=1,2$. Let $\Omega_{i}$, $i=1,2$, be the bounded, simply connected domains in $R^{2}$ with boundaries given by $\gamma_{i}, i=1,2$, respectively. By definition $\Omega_{2} \supset \Omega_{1} \supset \bigcup_{\alpha} \Omega_{\alpha}$.

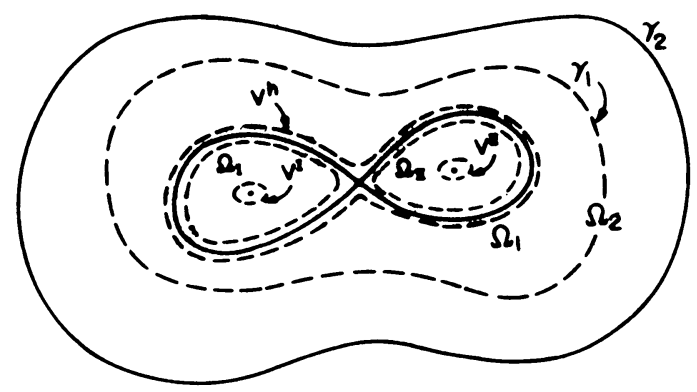

FIGURE 2

Let $K \subset \mathbf{R}^{2}$, a compact set, and choose $J_{1}$ for which $K \subset \operatorname{int}\left(\Omega_{1}\right)$. Let

$$
B_{K}=\left\{\varphi \in C_{0}^{\infty}\left(\Omega_{2}\right),\|\varphi\|_{L_{2}}=1, \operatorname{supp} \varphi \subseteq K\right\} .
$$

For each $\varepsilon>0$ we define $\mu^{\varepsilon}$ as the stationary measure due to the process (1.3), normalized by $\int_{\Omega_{1}} d \mu^{\varepsilon}=1$, and $\rho^{\varepsilon}$ is the associated density on $\Omega_{1}$.

By (1.17)-(1.20) we may present $\mu^{\varepsilon}$ on $\Omega_{1}$ by

$$
\int_{\Omega_{1}} \varphi d \mu^{\varepsilon}=\int_{\gamma_{1}} U_{\varphi}^{\varepsilon}(y) d \nu^{\varepsilon}(y) / \int_{\gamma_{1}} U_{1}^{\varepsilon}(y) d \nu^{\varepsilon}(y)
$$


where $U_{\varphi}^{\varepsilon}\left(U_{1}^{\varepsilon}\right)$ solves the Dirichlet problem (1.20) on $\Omega_{2}$ with r.h.s. given by $\varphi \in$ $B_{K}, 1_{\Omega_{1}}$, respectively.

We restrict ourselves, from now on, to the special case of Assumption B where the phase space includes a single homoclinic orbit (see Figure 1a). The proof in the general case is a direct generalization of this special case.

For each $\varphi \in L^{K}$ we define $U_{\varphi}^{0}$ on $\Omega_{2}$ as follows:

(a) $U_{\varphi}^{0}=U_{\varphi}^{0}(J)$ on each domain of oscillations $\Omega_{\alpha}, \alpha=I, I I$, and $\Omega^{e x} \cap \Omega_{2}$. It is smooth on each of the above domains.

(b) On each domain of oscillation, $U_{\varphi}^{0}$ satisfies

$$
\left\langle L^{\varepsilon} U_{\varphi}^{0}\right\rangle=-\langle\varphi\rangle \text {. }
$$

where the average $\langle\cdot\rangle$ is defined by $(2.14)$.

(c) $U_{\varphi}^{0}$ is a continuous function on $\Omega_{2}$.

(d) The total flux of $U_{\varphi}^{0}$ on the homoclinic orbit is zero, i.e.

$$
\int_{\partial \Omega_{I}} \frac{\partial}{\partial n} U_{\varphi}^{0}+\int_{\partial \Omega_{I I}} \frac{\partial}{\partial n} U_{\varphi}^{0}+\int_{\partial \Omega^{e x}} \frac{\partial}{\partial n} U_{\varphi}^{0}=0
$$

where $n$ is the outward normal vector to each of the above domains. By (a) above and the area interpretation of $J$ (see (2.3)), (3.3) is rewritten, using $\left(2.4^{\prime}\right)$, as

$$
\left.\left\langle\left|\nabla J^{I}\right|^{2}\right\rangle_{\bar{J}_{I}} \frac{d}{d J} U_{\varphi}^{0}\right|_{\bar{J}_{I}}+\left.\left\langle\left|\nabla J^{I I}\right|^{2}\right\rangle_{\bar{J}^{I I}} \frac{d}{d J} U_{\varphi}^{0}\right|_{\bar{J}_{I I}}=\left.\left\langle\left|\nabla J^{e x}\right|^{2}\right\rangle_{\bar{J}^{e x}} \frac{d}{d J} U_{\varphi}^{0}\right|_{\bar{J}^{e x}}
$$

(e) $\left.U_{\varphi}^{0}\right|_{J=J_{2}}=0$.

We define $U_{1}^{0}$ by conditions (a)-(e) where $\langle\varphi\rangle$ is replaced by $1_{\Omega_{1}}$ in (b).

LEMMA 3.1. There exists a unique $U_{\varphi}^{0},\left(U_{1}^{0}\right)$, satisfying $(\mathrm{a})-(\mathrm{e})$. If $\rho^{0}$ is given by $(2.16)$, normalized by $\iint_{\Omega_{1}} \rho^{0}=1$, then

$$
\int_{\Omega_{1}} \rho^{0} \varphi=U_{\varphi}^{0}\left(J_{1}\right) / U_{1}^{0}\left(J_{1}\right), \quad \forall \varphi \in l^{K} .
$$

PROOF. We prove existence and uniqueness by construction. From $(a, b)$ and some manipulations we obtain, on each domain of oscillation (see (3.20) in [12])

$$
\frac{d}{d J} U_{\varphi}^{0}=\frac{C}{\left\langle|\nabla J|^{2}\right\rangle} e^{-\chi(J)}+\frac{e^{-\chi(J)}}{\left\langle|\nabla J|^{2}\right\rangle} \int_{\beta}^{J} e^{\chi(s)}\langle\varphi\rangle_{(s)} d s
$$

for arbitrary constants $\beta, C$ and $\chi=\chi(J)$ defined by (2.12) separately on each domain. From (3.5) we obtain the flux due to $U_{\varphi}^{0}$ on each contour $\gamma_{s}$ in any of the domains

$$
\int_{\gamma_{s}} \frac{\partial}{\partial n} U_{\varphi}^{0}=\left.\left\langle|\nabla J|^{2}\right\rangle_{s} \frac{d}{d J} U_{\varphi}^{0}\right|_{J=s}=C e^{-\chi(s)}+e^{-\chi(s)} \int_{0}^{s} e^{\chi(u)}\langle\varphi\rangle_{(u)} d u .
$$

Consider the bounded domains of oscillations $\Omega_{\alpha}, \alpha=I, I I$. By (a), $C=0$, so

$$
\int_{\gamma_{\bar{J}_{I}}} \frac{\partial}{\partial n} U_{\varphi}^{0}+\int_{\gamma_{\bar{J}_{I}}} \frac{\partial}{\partial n} U_{\varphi}^{0}=\int_{0}^{\bar{J}_{I}} e^{\chi(u)}\langle\varphi\rangle_{(u)} d u+\int_{0}^{\bar{J}_{I I}} e^{\chi(u)}\langle\varphi\rangle_{(u)} d u .
$$


Notice that, by (2.12), $\chi\left(\bar{J}_{\alpha}\right)=\chi\left(\bar{J}^{e x}\right)=0, \alpha=I, I I$.

Consider now the external domain $\Omega^{e x}$. Letting $\beta=\bar{J}^{e x}$ in (3.5), $C$ is determined by (3.7) due to (d) and the remark above. Hence for $J=J^{e x}$

$$
\begin{array}{r}
\frac{d}{d J} U_{\varphi}^{0}=\frac{e^{-\chi(J)}}{\left\langle|\nabla J|^{2}\right\rangle}\left[\int_{0}^{\bar{J}_{I}} e^{\chi(u)}\langle\varphi\rangle_{u} d u+\int_{0}^{\bar{J}_{I I}} e^{\chi(u)}\langle\varphi\rangle_{u} d u+\int_{\bar{J}^{e x}}^{J} e^{\chi(u)}\langle\varphi\rangle_{u} d u\right] \\
J=J^{e x} \geq \bar{J}^{e x}
\end{array}
$$

while, for $J=J_{\alpha}$ in any of the bounded domains $\Omega_{\alpha}$ :

$$
\frac{d}{d J} U_{\varphi}^{0}=\frac{e^{-\chi(J)}}{\left\langle|\nabla J|^{2}\right\rangle} \int_{0}^{J} e^{\chi(u)}\langle\varphi\rangle(u) d u, \quad J=J_{\alpha} \leq \bar{J}_{\alpha} .
$$

Combining (3.8) and (3.9) and using the area interpretation of $J(2.3)$, we may rewrite both (3.8) and (3.9) as

$$
\frac{d}{d J} U_{\varphi}^{0}=\frac{e^{-\chi(J)}}{\left\langle|\nabla J|^{2}\right\rangle} \iint_{\{z ; J(z) \leq J\}} e^{\chi\left(z^{\prime}\right)}\langle\varphi\rangle\left(z^{\prime}\right) d z^{\prime} d \bar{z}^{\prime}, \quad 0 \leq J \leq J_{2} .
$$

Using (3.10), (c) and (e), we conclude

$$
U_{\varphi}^{0}(J)=-\int_{J}^{J_{2}} e^{-\chi(s)}\left\langle|\nabla J|^{2}\right\rangle_{s}^{-1}\left(\int_{0}^{s} e^{\chi(u)}\langle\varphi\rangle_{(u)} d u\right) d s .
$$

$U_{\varphi}^{0}$ satisfies conditions (a)-(e) and is unique by construction. Similarly, $U_{1}^{0}$ is uniquely defined by $(3.11)$ with $\langle\varphi\rangle$ replaced by $1_{\Omega_{1}}$. (3.11) can be written as

$$
U_{\varphi}^{0}(J)=\int_{0}^{J_{2}} \mathscr{K}(u) e^{\chi(u)}\langle\varphi\rangle_{(u)} d u
$$

where

$$
\mathscr{K}(u)=-\int_{J_{1}}^{J_{2}} 1(u \leq s)\left|\left\langle|\nabla J|^{2}\right\rangle\right|_{(s)}^{-1} e^{-\chi(s)} d s .
$$

Since $\operatorname{supp}\langle\varphi\rangle \subseteq K \subset \Omega_{1}$ and, by $(3.13), \mathscr{K}(u)=\mathscr{K}\left(J_{1}\right)$ for $0 \leq u \leq J_{1}$, we conclude from (3.12)

$$
\begin{aligned}
U_{\varphi}^{0}\left(J_{1}\right) & =\mathscr{K}\left(J_{1}\right) \int_{0}^{J_{1}} e^{\chi(u)}\langle\varphi\rangle_{(u)} d u \\
& =\mathscr{K}\left(J_{1}\right) \iint_{\{z ; J(z) \leq J\}} e^{\chi} \varphi d z d \bar{z} .
\end{aligned}
$$

On the other hand, letting $\varphi=1_{\Omega_{1}}$ in (3.11) and comparing to (3.14), we obtain

$$
U_{1}^{0}\left(J_{1}\right)=\mathscr{K}\left(J_{1}\right) \int_{0}^{J_{1}} e^{\chi(u)} d u
$$

and (3.4) follows by (3.14), (3.15).

The proof of Theorem 2.1 is now a direct consequence of the following lemma: 
LEMMA 3.2. Under the assumptions of Theorem 2.1

$$
\lim _{\varepsilon \rightarrow 0} \sup _{y \in \gamma_{1}}\left|U_{\varphi}^{\varepsilon}(y)-U_{\varphi}^{0}\left(J_{1}\right)\right|=0
$$

holds uniformly for $\varphi \in B_{K}$ and $\varphi=1_{\Omega_{1}}$.

Indeed, we obtain weak convergence of the $\Omega_{1}$ normalized distributions $\mu^{\varepsilon}$ by (3.1) and by the fact that $\nu^{\varepsilon}$ are probability distributions on $\gamma_{1}$. By (3.4) the above limit is given by $d \mu^{0}=\varphi^{0} d \mu^{L}$. Since the convergence in (3.16) is uniform w.r. to $\varphi \in B_{K}$, we also obtain the strong $L_{2}\left(\Omega_{1}\right)$ convergence for the associated densities.

Lemma 3.2 is a consequence of the following lemmas.

LEMMA 3.3. (a) For each periodic orbit $\gamma$ of the unperturbed system (1.1) (i.e. for each level curve of $H$ not containing critical points) there exists a constant $C(\gamma)<\infty$, independent of $\varepsilon$, and $\varphi \in B_{K}$, where

$$
\operatorname{osc}_{\gamma} U_{\varphi}^{\varepsilon} \leq \varepsilon^{1 / 4} C(\gamma)\left(1+\left\|\nabla U_{\varphi}^{\varepsilon}\right\|_{L_{2}}\right) .
$$

(b) Let $z \in \Omega_{2}, \bar{\nabla} H(z) \neq 0$. Then there exist a $\delta$ neighborhood of $z, V_{\delta}(z)$, and constants $C_{1}(z), C_{2}(z)$ independent of $\varepsilon$ and $\varphi \in B_{K}$, such that, for any $x, y \in V_{\delta}(z)$

$$
\left|U_{\varphi}^{\varepsilon}(x)-U_{\varphi}^{\varepsilon}(y)\right| \leq\left[C_{1}(z)|x-y|^{1 / 2}+\varepsilon^{1 / 4} C_{2}(z)\right]\left(1+\left\|\nabla U_{\varphi}^{\varepsilon}\right\|_{L_{2}}\right) .
$$

LEMMA 3.4. There exists $C$ independent of $\varepsilon$ and $\varphi \in B_{K}$, where

$$
\left\|\nabla U_{\varphi}^{\varepsilon}\right\|_{L_{2}\left(\Omega_{2}\right)} \leq C
$$

provided $\varepsilon$ is small enough.

Given $\delta>0$, consider the neighborhoods of the centers and the homoclinic orbits defined by

$$
\begin{gathered}
V^{\alpha}=\left\{J_{\alpha} ; J_{\alpha} \leq \delta\right\}, \quad \alpha=I, I I \\
V^{h}=\bigcup_{\alpha=I, I I}\left\{J_{\alpha} ; 0 \leq \bar{J}_{\alpha}-J_{\alpha} \leq \delta\right\} \cup\left\{J^{e x} ; \bar{J}^{e x} \leq J^{e x} \leq \bar{J}^{e x}+\delta\right\} .
\end{gathered}
$$

Define

$$
\begin{aligned}
& U_{\varphi}^{0, \delta}(J)=U_{\varphi}^{0}(J) \quad \text { for } J=J^{e x} \in\left(\Omega^{e x} \backslash V^{h}\right) \cap \Omega_{2}, \\
& U_{\varphi}^{0, \delta}(J)=\left.U_{\varphi}^{0}\right|_{J=J^{e x}=\bar{J}^{e x}+\delta \quad \text { for } J \in V^{h},} \\
& U_{\varphi}^{0, \delta}(J)=U_{\varphi}^{0}(J)+\left.U_{\varphi}^{0, \delta}\right|_{J \in V^{h}}-\left.U_{\varphi}^{0}\right|_{J=\bar{J}_{\alpha}-\delta} \text { on } \Omega_{\alpha} \backslash V^{\alpha}, \\
& U_{\varphi}^{0, \delta}(J)=\left.U_{\varphi}^{0, \delta}\right|_{J=J_{\alpha}=\bar{J}_{\alpha}} \text { on } V^{\alpha} .
\end{aligned}
$$

Notice that $U_{\varphi}^{0, \delta}$ is a continuous function on $\Omega_{2}$ and smooth on

$$
\Omega_{2} \backslash\left(\left[\bigcup_{\alpha=I, I I} \partial V^{\alpha}\right] \cup \partial V^{h}\right) .
$$

Moreover, on each $V^{\alpha}, \alpha=I, I I$ and $V^{h}, U_{\varphi}^{0, \delta}$ is a constant. Moreover, $U_{\varphi}^{0, \delta}\left(J_{2}\right)=$ $U_{\varphi}^{0}\left(J_{2}\right)=0$ (see Figure 2). 
LEMMA 3.5. There exist $C(\delta), g(\delta)$ independent of $\varepsilon, \varphi$

$$
\lim _{\delta \rightarrow 0} g(\delta)=0
$$

where

$$
\left\|\nabla U_{\varphi}^{\varepsilon}-\nabla U_{\varphi}^{0, \delta}\right\|_{L_{2}\left(\Omega_{2}\right)} \leq \varepsilon^{1 / 4} C(\delta)+g(\delta) .
$$

The proof of Lemma 3.2 follows directly from (3.17), (3.19) and (3.20). In fact, the r.h.s. of (3.20) is arbitrarily small for a proper choice of $\delta$ and $\varepsilon$. Since $U_{\varphi}^{\varepsilon}-U_{\varphi}^{0, \delta}$ satisfies the homogeneous boundary condition on $\gamma_{1}$ by the remark above, we can estimate $U_{\varphi}^{\varepsilon}-U_{\varphi}^{0, \delta}$, restricted to $\gamma_{1}$, as arbitrarily small in $L_{p}\left(\gamma_{1}\right), 1 \leq p<\infty$. By (3.17) and (3.19) the last estimate can be extended to $p=\infty$ and Lemma 3.2 follows.

PROOF OF LEMMA 3.3. We define $\partial_{H}$ as in $\S 1$. $U_{\varphi}^{\varepsilon}$ satisfies the inequalities:

$$
\begin{gathered}
\left\|\partial_{H} U_{\varphi}^{\varepsilon}\right\|_{L_{2}} \leq \varepsilon^{1 / 2} C\left(1+\left\|\nabla U_{\varphi}^{\varepsilon}\right\|_{L_{2}}\right), \\
\left\|\nabla \partial_{H} U_{\varphi}^{\varepsilon}\right\|_{L_{2}} \leq C\left(1+\left\|\nabla U_{\varphi}^{\varepsilon}\right\|_{L_{2}}\right),
\end{gathered}
$$

where $C$ is a constant independent of $\varepsilon$ and $\varphi \in B_{K}$. We will introduce the proofs of (3.21) and (3.22) later on.

Let $z_{0} \in \Omega_{2}, \nabla H\left(z_{0}\right) \neq 0$. On a small neighborhood $V\left(z_{0}\right)$ of $z_{0}$ we may define local coordinates $(s, t)$ and the associated mapping: $q(s, t) \in V\left(z_{0}\right)$ subject to

$$
\left.\frac{\partial q}{\partial t}\right|_{s, t}=\bar{\nabla} H(q(s, t)) \text {. }
$$

We may assume that $V\left(z_{0}\right)$ contains the rectangle $q\{-T \leq t \leq T,-\bar{s} \leq s \leq \bar{s}\}$. Define

$$
\chi(s)=U_{\varphi}^{\varepsilon}(q(s, 0))-U_{\varphi}^{\varepsilon}(q(s, T)) .
$$

Then, for $s_{1}, s_{2} \in[-\bar{s}, \bar{s}]$

$$
\begin{aligned}
\left|\int_{s_{1}}^{s_{2}} \chi(s) d s\right| & =\left|\int_{s_{1}}^{s_{2}} \int_{0}^{T} \frac{\partial}{\partial t} U_{\varphi}^{\varepsilon}(q(s, t)) d t d s\right| \\
& =\left|\int_{0}^{\bar{s}} \int_{0}^{T} \partial_{H} U_{\varphi}^{\varepsilon}(q(s, t)) d t d s\right| \\
& \leq T^{1 / 2}\left|s_{1}-s_{2}\right|^{1 / 2}\left(\int_{-\bar{s}}^{\bar{s}} \int_{-T}^{T}\left|\partial_{H} U_{\varphi}^{\varepsilon}\right|^{2} d t d s\right)^{1 / 2}
\end{aligned}
$$

where we used (3.23) in the second equality above. By (3.21) we estimate

$$
\left|\int_{s_{1}}^{s_{2}} \chi(s) d s\right| \leq T^{1 / 2}\left|s_{1}-s_{2}\right|^{1 / 2} \varepsilon^{1 / 2} C\left(1+\left\|\nabla U_{\varphi}^{\varepsilon}\right\|_{L_{2}}\right) .
$$

Next, consider

$$
\begin{aligned}
\left|\chi\left(s_{1}\right)-\chi\left(s_{2}\right)\right| & =\left|\int_{s_{1}}^{s_{2}} \int_{0}^{T} \frac{\partial}{\partial s} \frac{\partial}{\partial t} U_{\varphi}^{\varepsilon}(q(s, t)) d s d t\right| \\
& \leq\left|s_{1}-s_{2}\right|^{1 / 2} T^{1 / 2}\left[\int_{-\bar{s}}^{\bar{s}} \int_{-T}^{T}\left|\frac{\partial}{\partial s} \frac{\partial}{\partial t} U_{\varphi}^{\varepsilon}(q(s, t))\right|^{2} d s d t\right]^{1 / 2} .
\end{aligned}
$$


Changing variables and using (3.22),

$$
\left|\chi\left(s_{1}\right)-\chi\left(s_{2}\right)\right| \leq\left|s_{1}-s_{2}\right|^{1 / 2} T^{1 / 2} C \max _{V\left(z_{0}\right)}\left|\frac{\partial q_{1} \partial q_{2}}{\partial s \partial t}\right|^{-1}\left(1+\left\|\nabla U_{\varphi}^{\varepsilon}\right\|_{L_{2}}\right) .
$$

From (3.27)

$$
\inf _{-\bar{s} \leq s \leq \bar{s}} \chi(s) \geq \chi(0)-(2 \bar{s})^{1 / 2} T^{1 / 2} C\left(z_{0}\right)\left(1+\left\|\nabla U_{\varphi}^{\varepsilon}\right\|_{L_{2}}\right) .
$$

Hence, by (3.26) and a simple manipulation

$$
\chi(0) \leq C\left(z_{0}\right) T^{1 / 2}\left(1+\left\|\nabla U_{\varphi}^{\varepsilon}\right\|_{L_{2}}\right)\left[\varepsilon^{1 / 2}(2 \bar{s})^{-1 / 2}+(2 \bar{s})^{1 / 2}\right] .
$$

Setting $2 \bar{s}=\varepsilon^{1 / 2}$

$$
\chi(0) \leq \varepsilon^{1 / 4} C\left(z_{0}\right)\left(1+\left\|\nabla U_{\varphi}^{\varepsilon}\right\|_{L_{2}}\right) .
$$

Given $\gamma$ as in Lemma 3.3(a) we use a finite covering of $\gamma$ and (3.28) to obtain (3.17). In order to prove (3.18), notice that

$$
\begin{aligned}
& \mid U_{\varphi}^{\varepsilon}\left(q\left(s_{1}, t_{1}\right)\right)-U_{\varphi}^{\varepsilon}\left(q\left(s_{2}, t_{2}\right)\right) \\
& \quad \leq T^{-1}\left|\chi\left(s_{1}\right)-\chi\left(s_{2}\right)\right|+\sum_{i=1,2}\left|U_{\varphi}^{\varepsilon}\left(q\left(s_{i}, t_{i}\right)\right)-T^{-1} \chi\left(s_{i}\right)\right|
\end{aligned}
$$

The first term in (3.29) is estimated by (3.26) and the second by (3.17). (3.18) is now obtained by transforming back to the original coordinates.

We turn now to the proof of (3.21)-(3.22). Consider (1.20) in the weak form

$$
-\int_{\Omega_{2}} \nabla U_{\varphi}^{\varepsilon} \cdot \nabla V+\frac{1}{\varepsilon} \int_{\Omega_{2}} V \partial_{H} U_{\varphi}^{\varepsilon}+\int_{\Omega_{2}} V Q \cdot \nabla U_{\varphi}^{\varepsilon}=\int_{\Omega_{2}} \varphi V
$$

$V \in H_{0}^{1}\left(\Omega_{2}\right)$. Since $\partial \Omega_{2}=\gamma_{2}$ is a level curve of $H$ and all the coefficients are smooth, we may substitute $V=\partial_{H} U_{\varphi}^{\varepsilon}$ in (3.30). This yields

$$
\begin{aligned}
\frac{1}{\varepsilon} \int_{\Omega_{2}}\left|\partial_{H} U_{\varphi}^{\varepsilon}\right|^{2}= & \int_{\Omega_{2}} \varphi \partial_{H} U_{\varphi}^{\varepsilon}-\int_{\Omega_{2}}\left(\partial_{H} U_{\varphi}^{\varepsilon}\right) Q \cdot \nabla U_{\varphi}^{\varepsilon} \\
& +\int_{\Omega_{2}} \nabla U_{\varphi}^{\varepsilon} \cdot \nabla \partial_{H} U_{\varphi}^{\varepsilon}
\end{aligned}
$$

Using the identity

$$
\nabla \partial_{H}=\partial_{H} \nabla-\left[\partial_{H_{2}}, \partial_{H_{2}}\right], \quad H_{i}=\partial H / \partial x_{i}
$$

where $[\cdot, \cdot]$ stands for the Lie brackets, we evaluate the last term in $(3.30)$ by

$$
\int_{\Omega_{2}} \nabla U_{\varphi}^{\varepsilon} \partial_{H} \nabla U_{\varphi}^{\varepsilon}-\int_{\Omega_{2}} \nabla U_{\varphi}^{\varepsilon} \cdot\left[\partial_{H_{1}}, \partial_{H_{2}}\right] U_{\varphi}^{\varepsilon} .
$$

The first term in (3.32) is identically zero, while the second term is estimated by $C\left\|\nabla U_{\varphi}^{\varepsilon}\right\|_{L_{2}}^{2}$. Using the above estimate, the Cauchy-Schwarz inequality and the assumption $\|\varphi\|_{L_{2}} \leq 1$ due to the definition of $B_{K},(3.30)$ yields

$$
(1 / \varepsilon)\left\|\partial_{H} U_{\varphi}^{\varepsilon}\right\|_{L_{2}}^{2} \leq \bar{C}\left[\left\|\nabla U_{\varphi}^{\varepsilon}\right\|_{L_{2}}^{2}+\left\|U_{\varphi}^{\varepsilon}\right\|_{L_{2}}+\left\|\partial_{H} U_{\varphi}^{\varepsilon}\right\|_{L_{2}}\right]
$$


and (3.21) follows. To prove (3.22), substitute $U=\partial_{H}^{2} U_{\varphi}^{\varepsilon}$ in (3.30). (Again, this is a legitimate test function.) Thus we get

$$
\begin{aligned}
\int_{\Omega_{2}} \nabla U_{\varphi} \cdot \nabla \partial_{H}^{2} U_{\varphi}^{\varepsilon}= & -\int_{\Omega_{2}} \varphi \partial_{H}^{2} U_{\varphi}^{\varepsilon}+\int_{\Omega_{2}}\left(\partial_{H}^{2} U_{\varphi}^{\varepsilon}\right) Q \cdot \nabla U_{\varphi}^{\varepsilon} \\
& +\frac{1}{\varepsilon} \int_{\Omega_{2}}\left(\partial_{H} U_{\varphi}^{\varepsilon}\right) \partial_{H}^{2} U_{\varphi}^{\varepsilon}
\end{aligned}
$$

Notice that the last term on the r.h.s. of (3.33) is identically zero. Applying twice the identity $(3.31)$ on the l.h.s. of $(3.33)$ we obtain

$$
\int_{\Omega_{2}} \nabla U_{\varphi}^{\varepsilon} \cdot \nabla \partial_{H}^{2} U_{\varphi}^{\varepsilon}=\int_{\Omega_{2}} \nabla U_{\varphi}^{\varepsilon} \cdot \partial_{H}\left(\nabla \partial_{H} U_{\varphi}^{\varepsilon}\right)-\int_{\Omega_{2}} \nabla U_{\varphi}^{\varepsilon} \cdot\left[\partial_{H_{1}}, \partial_{H_{2}}\right] \nabla U_{\varphi}^{\varepsilon}
$$

and

$$
\int_{\Omega_{2}} \nabla U_{\varphi}^{\varepsilon} \cdot \partial_{H}\left(\nabla \partial_{H} U_{\varphi}^{\varepsilon}\right)=\int_{\Omega_{2}}\left|\nabla \partial_{H} U_{\varphi}^{\varepsilon}\right|^{2}-\int_{\Omega_{2}}\left[\partial_{H_{1}}, \partial_{H_{2}}\right] U_{\varphi}^{\varepsilon} \cdot \nabla \partial_{H} U_{\varphi}^{\varepsilon}
$$

A substitution of (3.34)-(3.35) in (3.33) and an application of the Cauchy-Schwarz inequality, together with the condition $\varphi \in B_{K}$, yields (3.22).

PROOF OF LEMMA 3.4. Let $\mu$ be a measure on $\Omega_{2}$, given by a continuous density $d \mu=\rho d \mu^{L}, \rho>0$. Assume, moreover, that $\rho$ admits a first derivative jump across a closed contour $\gamma \subset \Omega_{2}$, and smooth on $\Omega_{2} \backslash \gamma$. For any $U \in C^{2}\left(\Omega_{2}\right)$, $\left.U\right|_{\partial \Omega_{2}}=0$ define the quadratic form

$$
B_{\rho}(U, U) \equiv \int \rho U L^{\varepsilon} U=-\int_{\Omega_{2}} \rho|\nabla U|^{2}+\frac{1}{2} \int_{\Omega_{2} \backslash \gamma} U^{2}\left(L^{\varepsilon}\right)^{*} \rho-\frac{1}{2} \oint_{\gamma} U^{2}\left[\frac{\partial \rho}{\partial n}\right],
$$

where $L^{\varepsilon}$ as given in (1.4). The last term in (3.36) is a boundary integral which comes out due to the integration by parts. $[\partial \rho / \partial n]$ is the jump of the derivative of $\rho$ normal to $\gamma$, across $\gamma$. Let $\rho^{0}$ be given by (2.13). Then it follows, as expected

$$
\left(L^{\varepsilon}\right)^{*} \rho^{0}=\xi \quad \text { on } \Omega_{2} \backslash \partial \Omega^{e x}, \quad\langle\xi\rangle=0 .
$$

Evidently, $\xi$ is independent of $\varepsilon$ since $\rho^{0}=\rho^{0}(J)$, and is bounded on any compact in $\Omega_{2} \backslash \partial \Omega^{e x}$. Define $\rho^{0, \delta}$ by the same role as $U_{\varphi}^{0, \delta}$, i.e., $\rho^{0, \delta}=\rho^{0}$ on $\Omega^{e x} \backslash V^{h}, \rho^{0, \delta}=$ $\left.\rho^{0}\right|_{J=J^{e x}=\bar{J}^{e x}+\delta}$ on $V^{h}, \rho^{0, \delta}=\rho^{0}+\left[{\frac{0}{J}{ }^{e x}+\delta}-\rho \frac{0}{J_{\alpha}-\delta}\right]$ on $\Omega^{\alpha} \backslash V^{\alpha}$ and $\rho^{0, \delta}=\rho^{0}(\delta)$ on $V^{\alpha}, \alpha=I, I I$. Thus, $\rho^{0, \delta}$ is continuous and admits a jump in the normal derivatives across $\partial V^{\alpha}, \alpha=I, I I$, and $\partial V^{h}$. Hence

$$
\begin{gathered}
\left(L^{\varepsilon}\right)^{*} \rho^{0, \delta}=\xi \quad \text { on }\left(\Omega^{e x} \cap \Omega_{2}\right) \backslash V^{h}, \\
\left(L^{\varepsilon}\right)^{*} \rho^{0, \delta}=-(\nabla \cdot Q) \rho^{0, \delta} \text { on } V^{h}, \\
\left(L^{\varepsilon}\right)^{*} \rho^{0, \delta}=\xi-(\nabla \cdot Q) \Delta_{\delta}^{\alpha} \quad \text { on } \Omega_{\alpha} \backslash\left(V^{h} \cup V^{\alpha}\right), \quad \alpha=I, I I,
\end{gathered}
$$

where

$$
\Delta_{\delta}^{\alpha}=\rho_{\left(\bar{J}^{e x}+\delta\right)}^{0}-\rho_{\left(\bar{J}_{\alpha}-\delta\right)}^{0}
$$

and $\left(L^{\varepsilon}\right)^{*} \rho^{0, \delta}=-(\nabla \cdot Q) \rho^{0, \delta}$ on $V^{\alpha}, \alpha=I, I I$. 
Substituting $\rho^{0, \delta}$ for $\rho, U_{\varphi}^{\varepsilon}$ for $U$ in (3.36) and using (1.20) we get (3.38)

$$
\begin{aligned}
\int_{\Omega_{2}} \rho^{0, \delta}\left|\nabla U_{\varphi}^{\varepsilon}\right|^{2}= & \int_{\Omega_{2}} \rho^{0, \delta} U_{\varphi}^{\varepsilon} \varphi+\frac{1}{2} \int_{\Omega_{2}}\left|U_{\varphi}^{\varepsilon}\right|^{2}\left(L^{\varepsilon}\right)^{*} \rho^{0, \delta} \\
& -\frac{1}{2} \sum_{\alpha=I, I} \oint_{\partial V^{\alpha}}\left|U_{\varphi}^{\varepsilon}\right|^{2}\left[\frac{\partial}{\partial n} \rho^{0, \delta}\right]-\frac{1}{2} \oint_{\partial V^{h}}\left|U_{\varphi}^{\varepsilon}\right|^{2}\left[\frac{\partial}{\partial n} \rho^{0, \delta}\right] .
\end{aligned}
$$

Let $0<m=\inf _{\Omega_{2}} \rho^{0, \delta}, M=\sup _{\Omega_{2}} \rho^{0, \delta}$. The l.h.s. of (3.38) is estimated by

$$
\int_{\Omega_{2}} \rho^{0, \delta}\left|\nabla U_{\varphi}^{\varepsilon}\right|^{2} \geq m\left\|\nabla U_{\varphi}^{\varepsilon}\right\|_{L_{2}}^{2} .
$$

We now estimate the r.h.s. of (3.38) term by term

A. Estimate on $\int_{\Omega_{2}} \rho^{0, \delta} U_{\varphi}^{\varepsilon} \varphi$

$$
\left|\int_{\Omega_{2}} \rho^{0, \delta} U_{\varphi}^{\varepsilon} \varphi\right| \leq M\left\|U_{\varphi}^{\varepsilon}\right\|_{L_{2}}
$$

by definition of $B_{K}$.

B. Estimate on

$$
\frac{1}{2} \int_{\Omega_{2}}\left|U_{\varphi}^{\varepsilon}\right|^{2}\left(L^{\varepsilon}\right)^{*} \rho^{0, \delta}
$$

We rewrite (3.41) as

$$
\begin{aligned}
& \frac{1}{2}\left\{\int_{\Omega^{e x} \cap\left(\Omega_{2} \backslash V^{h}\right)}\left|U_{\varphi}^{\varepsilon}\right|^{2} \xi-\int_{V^{h}} \rho^{0, \delta}\left|U_{\varphi}^{\varepsilon}\right|^{2} \nabla \cdot Q\right. \\
& +\sum_{\alpha=I, I I} \int_{\Omega_{\alpha} \backslash\left(V^{h} \cup V^{\alpha}\right)}\left|U_{\varphi}^{\varepsilon}\right|^{2}\left(\xi-\nabla \cdot Q \Delta_{\delta}^{\alpha}\right) \\
& \left.-\sum_{\alpha=I, I I} \int_{V^{\alpha}}\left|U_{\varphi}^{\varepsilon}\right|^{2} \rho^{0, \delta} \nabla \cdot Q\right\} .
\end{aligned}
$$

The first term in (3.42) is written as

$$
\frac{1}{2} \int_{\Omega^{e x} \cap\left(\Omega_{2} \backslash V^{h}\right)}\left\langle\left|U_{\varphi}^{\varepsilon}\right|^{2}\right\rangle \xi+\frac{1}{2} \int_{\Omega^{e x} \cap\left(\Omega_{2} \backslash V^{h}\right)}\left(\left|U_{\varphi}^{\varepsilon}\right|^{2}-\left\langle\left|U_{\varphi}^{\varepsilon}\right|^{2}\right) \xi .\right.
$$

The first term of (3.43) is identically zero since $\langle\xi\rangle=0$. By (3.17),

$$
\left.|| U_{\varphi}^{\varepsilon}\right|^{2}-\left\langle\left|U_{\varphi}^{\varepsilon}\right|^{2}\right\rangle\left|\leq \varepsilon^{1 / 4} C(\delta) \sup _{\Omega_{2} \backslash\left(V^{h} \cup V^{I} \cup V^{I I}\right)}\right| U_{\varphi}^{\varepsilon} \mid \cdot\left(1+\left\|\nabla U_{\varphi}^{\varepsilon}\right\|_{L_{2}}\right),
$$

where

$$
C(\delta)=\sup _{\gamma \subset \Omega_{2} \backslash\left(V^{h} \cup V^{I} \cup V^{I I}\right)} C(\gamma),
$$

$C(\gamma)$ defined in Lemma 4.3(a). Notice that $\Omega_{2} \backslash\left(V^{h} \cup V^{I} \cup V^{I I}\right)$ includes no critical points of $\nabla H$. From Lemma 4.3(b) we conclude that

$$
\sup _{V}\left|U_{\varphi}^{\varepsilon}\right| \leq C(V)\left(1+\left\|\nabla U_{\varphi}^{\varepsilon}\right\|_{L_{2}}\right)
$$


for any compact set $V \subset \Omega_{2}$, not including critical points of $\nabla H$. Let $C(V)=C(\delta)$ for $V=\Omega_{2} \backslash\left(V^{h} \cup V^{I} \cup V^{I I}\right)$. In addition, we may assume $\sup _{V}|\xi| \leq C(\delta)$. Combining (3.43)-(3.44) we estimate the second term of (3.43), hence the first term in (3.42), by

$$
\left.\frac{1}{2}\left|\int_{V}\right| U_{\varphi}^{\varepsilon}\right|^{2} \xi \mid \leq \varepsilon^{1 / 4} C^{3}(\delta)\left(1+\left\|\nabla U_{\varphi}^{\varepsilon}\right\|_{L_{2}}\right)^{2}
$$

The second term in (3.42) is estimated by

$$
\begin{aligned}
\left.\frac{1}{2}\left|\int_{V^{h}} \rho^{0, \delta}\right| U_{\varphi}^{\varepsilon}\right|^{2} \nabla \cdot Q \mid & \leq \mathscr{M} \sup _{\Omega_{2}} \mid \nabla \cdot Q\left\|\left[\mu^{L}\left(V^{h}\right)\right]^{1 / 2}\right\| U_{\varphi}^{\varepsilon} \|_{L_{4}}^{2} \\
& \leq \hat{\mathscr{M}}\left[\mu^{L}\left(V^{h}\right)\right]^{1 / 2}\left\|\nabla U_{\varphi}^{\varepsilon}\right\|_{L_{2}}^{2},
\end{aligned}
$$

independent of $\varepsilon, \delta$ and $\varphi$ and $\mu^{L}$ is a Lebesgue measure. In a way similar to (3.46)-(3.47), the last two terms in (3.42) are estimated by

$$
\begin{aligned}
\varepsilon^{1 / 4} C^{3}(\delta)\left(1+\left\|\nabla U_{\varphi}^{\varepsilon}\right\|_{L_{2}}\right)^{2} & +\left(\Delta_{\delta}^{I}+\Delta_{\delta}^{I I}\right) \hat{\mathscr{M}}\left\|\nabla U_{\varphi}^{\varepsilon}\right\|_{L_{2}}^{2} \\
& +\hat{\mathscr{M}}\left[\left(\mu^{L}\left(V^{I}\right)\right)^{1 / 2}+\left(\mu^{L}\left(V^{I I}\right)\right)^{1 / 2}\right]\left\|\nabla U_{\varphi}^{\varepsilon}\right\|_{L_{2}}^{2},
\end{aligned}
$$

which, together with (3.46)-(3.47) provides the estimate of $(3.41)\left(\Delta_{\delta}^{\alpha}\right.$ as defined in (3.37)).

C. Estimate on

$$
-\frac{1}{2} \oint_{\partial V^{\alpha}}\left|U_{\varphi}^{\varepsilon}\right|^{2}\left[(\partial / \partial n) \rho^{0, \delta}\right]
$$

By definition of $\rho^{0}$ and $\left(2.14^{\prime}\right),(3.49)$ is estimated by

$$
\left|\frac{d \rho^{0}}{d J}(\delta)\right|\left\langle\left|U_{\varphi}^{\varepsilon} \nabla J\right|^{2}\right\rangle_{(\delta)} \leq \frac{d \rho^{0}}{d J}(\delta) \sup _{\gamma_{\delta}}|\nabla J|^{2}\left\langle\left|U_{\varphi}^{\varepsilon}\right|^{2}\right\rangle_{(\delta)} .
$$

Given $J^{0}<\bar{J}_{\alpha}$, we obtain

$$
\begin{aligned}
\left\langle\left|U_{\varphi}^{\varepsilon}\right|^{2}\right\rangle_{J^{0}}-\left\langle\left|U_{\varphi}^{\varepsilon}\right|^{2}\right\rangle_{\delta} & =\frac{1}{2 \pi} \int_{0}^{2 \pi}\left(\left|U_{\varphi}^{\varepsilon}\right|^{2}\left(J^{0}, \theta\right)-\left|U_{\varphi}^{\varepsilon}\right|^{2}(\delta, \theta)\right) d \theta \\
& \leq \frac{1}{2 \pi} \int_{0}^{2 \pi} \int_{\delta}^{J^{0}}\left|U_{\varphi}^{\varepsilon} \frac{\partial}{\partial J} U_{\varphi}^{\varepsilon}\right|^{2}(s, \theta) d s d \theta
\end{aligned}
$$

where we used the $(J, \theta)$ coordinate representation of $U_{\varphi}^{\varepsilon}$. The last term above is estimated by

$$
\sup _{\delta \leq J \leq J^{0}}\left\{|\nabla J|^{-1}\right\}\left\|U_{\varphi}^{\varepsilon}\right\|_{L_{2}}\left\|\nabla U_{\varphi}^{\varepsilon}\right\|_{L_{2}}
$$

For $J=s \leq J_{0}$ small enough we use the assumption of nondegeneracy of the critical point at $J=0$ (Assumption B) to obtain

$$
C_{1} s^{1 / 2} \leq \inf _{J=s}|\nabla J| \leq \sup _{J=s}|\nabla J| \leq C_{2} s^{1 / 2},
$$

(see (2.6)), Using (3.50)-(3.53) we estimate (3.49) as

(3.54) $\left.\delta C_{2}^{2} \frac{d \rho^{0}}{d J}\right|_{(\delta)}\left[\left\langle\left|U_{\varphi}^{\varepsilon}\right|^{2}\right\rangle_{J^{0}}+C_{1} \delta^{-1 / 2}\left\|U_{\varphi}^{\varepsilon}\right\|_{L_{2}}\left\|\nabla U_{\varphi}^{\varepsilon}\right\|_{L_{2}}\right] \leq C \delta+C \delta^{1 / 2}\left\|\nabla U_{\varphi}^{\varepsilon}\right\|_{L_{2}}^{2}$ 
where $C$ is independent of $\delta, \varepsilon, \varphi \in B_{K}$. In (3.54) we used Lemma 3.3(b) to obtain an independent bound on $\left\langle\left|U_{\varphi}^{\varepsilon}\right|^{2}\right\rangle_{J^{0}}$.

D. Estimate on

$$
\frac{1}{2} \oint_{\partial V^{h}}(\partial / \partial n) \rho^{0, \delta}\left|U_{\varphi}^{\varepsilon}\right|^{2} .
$$

Since $\rho^{0, \delta}=$ const on $V^{h}, \rho^{0, \delta}=\rho^{0}$ up to a constant on $\Omega_{2} \backslash\left(V^{h} \cup V^{I} \cup V^{I I}\right),(3.55)$ is written as

$$
\frac{1}{2}\left[\oint_{\gamma^{e x}}\left|U_{\varphi}^{\varepsilon}\right|^{2} \nabla \rho^{0} \cdot n-\oint_{\gamma^{I}}\left|U_{\varphi}^{\varepsilon}\right|^{2} \nabla \rho^{0} \cdot n-\oint_{\gamma^{I I}}\left|U_{\varphi}^{\varepsilon}\right|^{2} \nabla \rho^{0} \cdot n\right],
$$

where

$$
\gamma^{e x}=\partial V^{h} \cap \Omega^{e x}, \quad \gamma^{\alpha}=\partial V^{h} \cap \Omega_{\alpha}, \quad \alpha=I, I I .
$$

Let

$$
d(\delta)=\max \left[\operatorname{dist}\left(\gamma^{e x}, \gamma^{I}\right), \operatorname{dist}\left(\gamma^{e x}, \gamma^{I I}\right)\right] .
$$

Given $z_{1}$ on the homoclinic orbit, $\nabla H\left(z_{1}\right) \neq 0$, and using both parts of Lemma 3.3 , we obtain

$$
\sup _{z \in \partial V^{h}}\left|U_{\varphi}^{\varepsilon}(z)-U_{\varphi}^{\varepsilon}\left(z_{1}\right)\right| \leq\left(\varepsilon^{1 / 4} C(\delta)+C d^{1 / 2}(\delta)\right)\left(1+\left\|\nabla U_{\varphi}^{\varepsilon}\right\|_{L_{2}}\right)
$$

where $C$ above is independent of $\varepsilon, \delta, \varphi \in B_{K}$. In addition, by Lemma 3.3(b),

$$
\left|U_{\varphi}^{\varepsilon}\left(z_{1}\right)\right| \leq C\left(1+\left\|\nabla U_{\varphi}^{\varepsilon}\right\|_{L_{2}}\right)
$$

since $z_{1}$ is fixed (independent of $\delta$ ).

Combining (3.56), (3.57), we estimate (3.55) by

$$
C\left\{\left|\int_{\partial V^{h}} \nabla \rho^{0} \cdot n\right|+\left(\varepsilon^{1 / 4} C(\delta)+C d^{1 / 2}(\delta)\right) \int_{\partial V^{h}}\left|\nabla \rho^{0} \cdot n\right|\right\}\left(1+\left\|\nabla U_{\varphi}^{\varepsilon}\right\|_{L_{2}}\right)^{2} .
$$

In the first term above $n$ is understood as the unit normal in the direction outside $V^{h}$. By (2.9), (2.12), (2.13) we obtain

$$
\begin{aligned}
\left|\int_{\partial V^{n}} \nabla \rho^{0} \cdot n\right| \leq & \rho^{0}\left(\bar{J}^{I}-\delta\right)\left|\langle Q \cdot \nabla J\rangle_{\bar{J}^{I}-\delta}+\langle Q \cdot \nabla J\rangle_{\bar{J}^{I I}-\delta}-\langle Q \cdot \nabla J\rangle_{\bar{J}^{e x}+\delta}\right| \\
& +\max _{\alpha=I, I I}\left(\left|\Delta_{\delta}^{I}\right|,\left|\Delta_{\delta}^{I I}\right|\right) \max \left|\left(\langle Q \cdot \nabla J\rangle_{\gamma} ; \gamma=\gamma^{I}, \gamma^{I I}, \gamma^{e x}\right)\right|
\end{aligned}
$$

where $\Delta_{\delta}^{\alpha}$ is defined as in (3.37).

Using the divergence theorem, the first term in (3.60) is written as

$$
\rho^{0}\left(\bar{J}^{I}-\delta\right) \iint_{V^{h}} \nabla \cdot Q \leq C \mu^{L}\left(V^{h}\right),
$$

and the second term of (3.60) is estimated by

$$
C \int_{\Omega_{2}}|\nabla \cdot Q| \cdot \max _{\alpha=I, I I}\left(\left|\Delta_{\delta}^{I}\right|,\left|\Delta_{\delta}^{I I}\right|\right) \leq C \max _{d=I, I I}\left(\left|\Delta_{\delta}^{I}\right|, \mid \Delta_{\delta}^{I I}\right) .
$$

Using (3.60)-(3.61), we estimate (3.59), hence (3.55), by

$$
C\left[\mu^{L}\left(V^{h}\right)+\max _{\alpha=I, I I}\left|\Delta_{\delta}^{\alpha}\right|+\varepsilon^{1 / 4} C(\delta) C d^{1 / 2}(\delta)\right]\left(1+\left\|\nabla U_{\varphi}^{\varepsilon}\right\|_{L_{2}}\right)^{2} .
$$


Substituting the estimates for $A, B, C$ and $D$ in the r.h.s., using (3.39) for the l.h.s. of $(3.38)$, we obtain

$$
\begin{aligned}
m\left\|\nabla U_{\varphi}^{\varepsilon}\right\|_{L_{2}}^{2} \leq & M\left\|U_{\varphi}^{\varepsilon}\right\|_{L_{2}}+C\left[C(\delta) \varepsilon^{1 / 4}+\left|\Delta_{\delta}^{I}\right|+\left|\Delta_{\delta}^{I I}\right|+\left(\mu^{L}\left(V^{I}\right)\right)^{1 / 2}\right. \\
& \left.+\left(\mu^{L}\left(V^{I I}\right)\right)^{1 / 2}+\mu^{L}\left(V^{h}\right)+d^{1 / 2}(\delta)+C \delta^{1 / 2}\right]\left(\left\|\nabla U_{\varphi}^{\varepsilon}\right\|_{L_{2}}+1\right)^{2}+C \delta .
\end{aligned}
$$

Notice that $\mu^{L}\left(V^{\alpha}\right), \mu^{L}\left(V^{h}\right) \Delta_{\delta}^{\alpha}(\alpha=I, I I)$ and $d(\delta)$ are arbitrarily small for $\delta$ small enough. Taking $\delta$ small enough, then $\varepsilon$ small enough in (3.64) we obtain

$$
\left\|\nabla U_{\varphi}^{\varepsilon}\right\|_{L_{2}}^{2} \leq \bar{C}_{1}\left\|U_{\varphi}^{\varepsilon}\right\|_{L_{2}}+g(\delta)
$$

$\bar{C}_{1}$ independent of $\varepsilon, \delta, \lim _{\delta \rightarrow 0} g(\delta)=0$, and $\varphi \in B_{K}$. (3.65) and the homogeneous boundary condition of $U_{\varphi}^{\varepsilon}$ yield (3.19).

PROOF OF LEMMA 3.5. Let $U_{\varphi}^{0, \delta}$ be as defined (see paragraph preceding Lemma 3.4) and $\rho^{0, \delta}$ be as given in the proof of Lemma 3.4. Let $W_{\varphi}^{\varepsilon, \delta}=U_{\varphi}^{\varepsilon}-U_{\varphi}^{0, \delta}$. Then, by definition

$$
\begin{gathered}
L^{\varepsilon} W_{\varphi}^{\varepsilon, \delta}=-\varphi+L^{\varepsilon} U_{\varphi}^{0} \quad \text { on } V, \quad V=\Omega_{2} \backslash\left(V^{I} \cup V^{I I} \cup V^{h}\right) \\
L^{\varepsilon} W_{\varphi}^{\varepsilon, \delta}=-\varphi \quad \text { on } \Omega_{2} \backslash V=V^{I} \cup V^{I I} \cup V^{h}
\end{gathered}
$$

and

$$
\int_{\Omega_{2}} \rho^{0, \delta} W_{\varphi}^{\varepsilon, \delta} L^{\varepsilon} W_{\varphi}^{\varepsilon, \delta}=\int_{\Omega_{2} \backslash V} \rho^{0, \delta}\left(-\varphi+L^{\varepsilon} U_{\varphi}^{0}\right) W_{\varphi}^{\varepsilon, \delta}+\frac{1}{2} \int_{V} \rho^{0, \delta} W_{\varphi}^{\varepsilon, \delta} \varphi .
$$

Integration by parts on the 1.h.s. of (3.66) yields

$$
\begin{aligned}
\int_{\Omega_{2}} \rho^{0, \delta}\left|\nabla W_{\varphi}^{\varepsilon, \delta}\right|^{2}= & \int_{\Omega_{2} \backslash V} \rho^{0, \delta}\left(-\varphi+L^{\varepsilon} U_{\varphi}^{0}\right) W_{\varphi}^{\varepsilon, \delta}+\frac{1}{2} \int_{V} \rho^{0, \delta} W_{\varphi}^{\varepsilon, \delta} \varphi \\
& +\frac{1}{2} \int_{\Omega_{2}}\left|W_{\varphi}^{\varepsilon, \delta}\right|^{2}\left(L^{\varepsilon}\right)^{*} \rho^{0, \delta} \\
& -\frac{1}{2} \int_{\partial V}\left|W_{\varphi}^{\varepsilon}\right|^{2}\left[\frac{\partial}{\partial n} \rho^{0, \delta}\right]-\frac{1}{2} \oint_{\partial V} \rho^{0, \delta} W_{\varphi}^{\varepsilon}\left[\frac{\partial}{\partial n W_{\rho}^{\varepsilon}}\right]
\end{aligned}
$$

The estimate on the r.h.s. of (3.67) is essentially the same as that of (3.38) in Lemma 3.4. In fact, $W_{\varphi}^{\varepsilon, \delta}$ satisfies, by its definition, the same a priori estimates as $U_{\varphi}^{\varepsilon}$ given by Lemma 3.3. There are only two points we have to consider:

(a) The first two first terms on the r.h.s. of (3.67) replace the first term on the right of $(3.38)$. Since $\left\langle-\varphi+L^{\varepsilon} U_{\varphi}^{0}\right\rangle=0$ on $\Omega_{2} \backslash V$, and $\rho^{0, \delta}=\rho^{0, \delta}(J)$ we may replace $W_{\varphi}^{\varepsilon, \delta}$ by $W_{\varphi}^{\varepsilon, \delta}-\left\langle W_{\varphi}^{\varepsilon, \delta}\right\rangle$ in the first term above. Thus we estimate this term by

$$
M\left\|-\varphi+L^{\varepsilon} U_{\varphi}^{0}\right\|_{L_{1}(V)} \sup _{V}\left|W_{\varphi}^{\varepsilon, \delta}-\left\langle W_{\varphi}^{\varepsilon, \delta}\right\rangle\right|,
$$

and $\left|W_{\varphi}^{\varepsilon, \delta}-\left\langle W_{\varphi}^{\varepsilon, \delta}\right\rangle\right|=\left|U_{\varphi}^{\varepsilon}-\left\langle U_{\varphi}^{\varepsilon}\right\rangle\right| \leq C \varepsilon^{1 / 4}$ on $V$ by Lemma 3.3(a) and Lemma 3.4. We only have to check $\left\|L^{\varepsilon} U_{\varphi}^{0}\right\|_{L_{1}(V)} \leq C(\delta)$ independently of $\varphi \in B_{K}$. This follows from the definition of $U_{\varphi}^{0}(3.11)$ and the inequality

$$
\|\langle\varphi\rangle\|_{L_{1}} \leq C\|\varphi\|_{L_{1}} \leq C^{\prime}\|\varphi\|_{L_{2}} \leq C^{\prime}, \quad \varphi \in B_{K}
$$

The second term on the right of (3.67) is estimated by

$$
\frac{1}{2} \mathscr{M}\left\|W_{\varphi}^{\varepsilon, \delta}\right\|_{L_{4}}\|\varphi\|_{L_{2}}\left(\mu^{L}(V)\right)^{1 / 2} \leq C\left\|\nabla W_{\varphi}^{\varepsilon, \delta}\right\|_{L_{2}}\left(\mu^{L}(V)\right)^{1 / 2}
$$


since $\|\varphi\|_{L_{2}} \leq 1$ and by the Sobolev inequality. Hence

$$
\int_{\Omega_{2}} \rho^{0, \delta}\left(-\varphi+L^{\varepsilon} W_{\varphi}^{\varepsilon, \delta}\right) \leq C(\delta) \varepsilon^{1 / 4}+C\left(\mu^{L}(V)\right)^{1 / 2}\left\|\nabla W_{\varphi}^{\varepsilon, \delta}\right\|_{L_{2}}
$$

replaces the estimate on $\int_{\Omega_{2}} \rho^{0, \delta} \varphi U_{\varphi}^{\varepsilon}$ in (3.40).

(b) The last term on the right of (3.67) is due to the jump in normal derivatives of $W_{\varphi}^{\varepsilon, \delta}$ across $\partial V$. It does not appear in (3.38) since $U_{\varphi}^{\varepsilon}$ is smooth. In fact,

$$
\oint_{\partial V} \rho^{0, \delta} W_{\varphi}^{\varepsilon, \delta} \frac{\partial}{\partial n}\left[W_{\varphi}^{\varepsilon, \delta}\right]=-\oint_{\partial V} \rho^{0, \delta} W_{\varphi}^{\varepsilon, \delta}\left[\frac{\partial}{\partial n} U_{\varphi}^{0, \delta}\right],
$$

and is estimated by an analysis similar to $C, D$ of the previous proof.

Note that $\left\|W_{\varphi}^{\varepsilon, \delta}\right\|_{L_{2}}$ admits the same estimate as $\left\|U_{\varphi}^{\varepsilon}\right\|_{L_{2}}$. The desired inequality (3.20) follows by collecting all the estimates, as in Lemma 3.4, where (3.40) is replaced by (3.69).

By the remarks following (3.16) and (3.20) we have completed the proofs of Theorem 2.1.

\section{REFERENCES}

1. V. I. Arnold, Mathematical methods of classical mechanics, Springer-Verlag, 1970.

2. M. Berger, Private communication.

3. R. N. Bhattacharya, Criteria for recurrence and existence of invariant measures for multidimensional diffusions, Ann. Probab. 6 (1978), 541-553.

4. A. Friedman, Stochastic differential equations and application, Vol. I, Academic Press, 1976.

5. E. Hille, and R. Phillips, Functional analysis and semigroups, rev. ed., Amer. Math. Soc., Providence, R.I., 1957.

6. R. Z. Khas'minskii, Ergodic properties of recurrent diffusion process and stabilization of the solution of the Cauchy problem for parabolic equations, Theory Probab. Appl. 5 (1960), 179191.

7. __ Principle of averaging for parabolic and elliptic differential equations and for Markov process with small diffusion, Theory. Probab. Appl. 7 (1963), 1-21.

8. _ The behavior of a self oscillating system acted upon by slight noise, Prikl. Mat. Mek. 27 (1963), 683-687.

9. The behavior of a conservative system under the action of a slight friction and slight random noise, 28 (1964), 931-935.

10. T. G. Kurtz, A limit theorem for perturbed operator semigroups with applications to random evolution, J. Funct. Anal. 12 (1973), 55-67.

11. R. Pinsky, Private communication.

12. G. Wolansky, Stochastic perturbations to conservative dynamical systems on the plane. II: Recurrency conditions, Trans. Amer. Math. Soc. 309 (1988), 641-657.

13. _ Elliptic perturbations of nonlinear oscillations in the presence of resonances, Indiana $\mathbf{J}$. Math. (to appear).

Department of Theoretical Mathematics, The Weizmann Institute of SCIENCE, REHOVOT 76100, ISRAEL 